\title{
Computing the Pessimism of Inclusion Functions
}

\author{
Gilles Chabert and Luc Jaulin \\ ENSIETA, 2 rue François Verny 29806 Brest Cedex 9 \\ gilles.chabert@ensieta.fr luc.jaulin@ensieta.fr
}

\begin{abstract}
Computing the pessimism" means bounding the overestimation produced by an inclusion function. There are two important distinctions with classical error analysis. First, we do not consider the image by an inclusion function but the distance between this image and the exact image (in the set-theoretical sense). Second, the bound is computed over a infinite set of intervals.

To our knowledge, this issue is not covered in the literature and may have a potential of applications. We first motivate and define the concept of pessimism. An algorithm is then provided for computing the pessimism, in the univariate case. This algorithm is general-purpose and works with any inclusion function. Next, we prove that the algorithm converges to the optimal bound under mild assumptions. Finally, we derive a second algorithm for automatically controlling the pessimism, i.e., determining where an inclusion function is accurate.
\end{abstract}

\section{Introduction}

In this paper, we consider a continuous function $f: \mathbb{R} \rightarrow \mathbb{R}$ (the definition domain of $f$ is assumed to be $\mathbb{R}$ for simplicity). The radius, the middle, the magnitude and the mignitude of an interval $[x]$ are denoted by rad $[x]$, mid $[x]$, $|[x]|$ and $<[x]>$ respectively.

One fundamental tool that interval analysis provides is the notion of inclusion function (see, e.g., $[10,5]$ ). An inclusion function $F$ is a mapping from $\mathbb{R} \mathbb{R}$ (the set of intervals) to $\mathbb{I} \mathbb{R}$ such that

$$
\forall[x] \in \mathbb{R} \quad f([x]) \subseteq F[x],
$$

where $f([x])$ denotes the set-theoretical image of $[x]$ by $f$.

An inclusion function allows to perform a safe evaluation of a function on a whole set of values and this addresses the question of reliability: thanks to inclusion functions, interval methods are robust to round-off errors, imprecision on input data, numerical truncatures, etc. Besides, the question of accuracy, i.e., whether the result is accurate or not, is also a crucial matter for numerical methods. Clearly, if the overestimation produced by the underlying inclusion function is really too large, the method will suffer from a lack of accuracy. 
The accuracy issue has led people to design smart inclusion functions using, e.g., multi-precision arithmetic [11], Taylor models [7] or Bernstein expansions for polynomials [4]. See also [10] for a survey on inclusion functions.

The purpose of this paper is to evaluate the accuracy of any given (inclusion isotone) inclusion function. This purpose can first be formulated as follows:

Problem 1. Given an inclusion function $F$ and an interval $[x]$, find a bound for

$$
\text { overestim }[x]:=\operatorname{rad} F[x]-\operatorname{rad} f([x]) .
$$

If we consider that a sophisticated inclusion function necessarily involves more computations, this problem becomes relevant since it is useful to know whether it is worth the effort or not.

\subsection{A first approach}

A first property of inclusion functions connected to accuracy is called Lipschitzcontinuity [9]. In particular, this property implies that the radius of $F[x]$ goes to zero with the radius of $[x]$, i.e., that the overestimation can be arbitrarily reduced by conveniently splitting the domains.

Definition 1 (Lipschitz-continuity of an inclusion function). An inclusion function $F$ is said to be Lipschitz-continuous if for all interval $[x]^{(0)} \in \mathbb{I} \mathbb{R}$, there exists a constant $\lambda_{F}\left(\left[x^{(0)}\right]\right.$ such that

$$
\forall[x],[y] \subseteq[x]^{(0)} \quad q(F[x], F[y]) \leq \lambda_{F}\left(\left[x^{(0)}\right]\right) q([x],[y])
$$

where $q$ is the Hausdorff distance:

$$
q([x],[y])=|\operatorname{mid}[x]-\operatorname{mid}[y]|+|\operatorname{rad}[x]-\operatorname{rad}[y]| .
$$

The easiest way to bound the overestimation for a given interval $[x]$ is to write:

$$
\operatorname{overestim}([x]) \leq \operatorname{rad} F[x] .
$$

This approximation makes sense if we assume that $[x]$ is a perturbed value mid $[x]$ by a small uncertainty rad $[x]$ and that $f$ is locally almost flat. In this way, the output error $(\operatorname{rad} f([x]))$ can be neglected so that the overestimation can be identified to the radius of $F[x]$. Under these conditions, the following proposition (see [9] Cor. 2.1.2) allows then to bound the overestimation:

Proposition 1. If $F$ is Lipschitz-continuous then

$$
\forall[x] \in \mathbb{I} \mathbb{R} \quad \operatorname{rad} F[x] \leq \lambda_{F}([x]) \operatorname{rad}[x] .
$$


However, this estimation is not adapted for set computations, i.e., for situations where $[x]$ does not describe a single perturbed value but a full range of possibilities. Indeed, in the latter case, there may be an important gap between the range of the overestimation and the range of $F$ itself. Furthermore, this estimation is relevant when intervals have an extremely small width (this is why we usually remember a linear rate of improvement from Proposition 1).

\section{$1.2 \quad$ A new approach}

No theoretical tool seems available for precisely computing the overestimation of an arbitrary inclusion function. This is not a big surprise since this quantity usually strongly depends on both the expression of the function ${ }^{1}$ and the actual arguments.

We may therefore turn to numerical means. Unfortunately, from the numerical standpoint, Problem 1 has little sense in general, since an accurate computation of overestim $[x]$ is likely to require more computations than an evaluation with the "best" inclusion function at our disposal.

Computing the overestimation can nevertheless be made meaningful with a slightly different point of view. Consider the two following facts:

- Due to the combinatorial effect of any branch \& bound algorithm (see e.g., [6, $12,5,2]$ ) functions are often repeatedly evaluated with overlapping domains. Therefore, spending some time for analyzing offline the behaviour of a given inclusion function $F$ may pay.

- We are more interested by checking that the inclusion function satisfies some precision requirements globally than by determining the overestimation for a specific interval.

Based on these observations, the problem of computing the overestimation of an inclusion function can be reformulated as follows:

Problem 2. Given an inclusion function $F$ and an interval $[x]^{(0)}$, is the overestimation of $F$ less than a user-defined threshold $\varepsilon$ for any $[x] \subseteq[x]^{(0)}$ ?

Note that the answer is not as simple as computing overestim $[x]$ and checking overestim $[x]<\varepsilon$. The reason is that the overestimation is not inclusion isotonic, i.e.,

$$
[y] \subseteq[x] \Longrightarrow \text { overestim }[y] \leq \text { overestim }[x] .
$$

This can be shown on a simple counterexample:

\footnotetext{
${ }^{1}$ The expression of the function comes into play providing that this expression is somehow involved in the definition of the inclusion function. As an example, consider the mean value form (see [9] p.51). If an enclosure of the derivative is computed with automatic differentiation then the overestimation depends on the expression of $f$.
} 
Example 1. Let $f: x \mapsto \sin (\pi x / 2)+x$ and consider the natural extension $F$ of $f$.

On the one hand, we have $F[-1,5]=\sin ([-\pi / 2,5 \pi / 2])+[-1,5]=[-2,6]$, which exactly matches $f([-1,5])$ since $f(-1)=-2$ and $f(5)=6$. Hence, overestim $[-1,5]=0$.

On the other hand, $F[1,2]=\sin ([\pi / 2, \pi])+[1,2]=[0,3]$ whereas $f([1,2]) \sim$ $[2.0,2.2]$.

Solving Problem 2 requires the ability to calculate the overestimation for a whole set of possible input intervals. For convenience, this maximal overestimation will be called the pessimism of $F$ in the sequel. Hence, the question amounts to : given an inclusion function $F$ and an interval $[x]$, is there an algorithm for computing

$$
\operatorname{pessim}[x]:=\max _{[y] \subseteq[x]} \text { overestim }[y] ?
$$

We propose in the two next sections such an algorithm. For the sake of clarity, it is split into two subalgorithms pessim ${ }^{+}$and pessim ${ }^{-}$that computes an upper and lower bound of the pessimism respectively; each of them being treated in a separate section. The convergence property of both subalgorithms will be stated.

We will introduce in Section 5 a more high-level algorithm for controlling the pessimism.

\section{Pessimism upper bound}

Starting now, we consider a fixed inclusion function $F$ that satisfies inclusion isotonicity, i.e., $[x] \subseteq[y] \Longrightarrow F[x] \subseteq F[y]$. We present in this section the pessim ${ }^{+}$algorithm that upwardly bounds (1), i.e., the maximal overestimation produced by $F$ on a given interval $[x]$.

First of all, the pessim ${ }^{+}$algorithm creates a uniform subdivision of $[x]$ by a sequence of $(n+1)$ points

$$
x_{1}=l b([x]) \quad, \ldots, \quad x_{i}=x_{1}+\frac{i-1}{n} \operatorname{rad}[x] \quad, \ldots, \quad x_{n+1}=u b([x]),
$$

where $l b([x])$ and $u b([x])$ stands for the lower and upper bound of $[x]$ respectively. The number $n$ is (besides $[x]$ ) a parameter of the pessim ${ }^{+}$algorithm. We will call a $k$-block an interval $\left[x_{i}, x_{i+k}\right]$ of the subdivision.

In addition, the pessim ${ }^{+}$algorithm computes an inner approximation of the image of $f$ on every $k$-block $(1 \leq k \leq n)$. We will denote by range ${ }^{-}\left(\left[x_{i}, x_{i+k}\right]\right)$ such approximation. In a word:

$$
\operatorname{range}^{-}\left(\left[x_{i}, x_{i+k}\right]\right) \subseteq f\left(\left[x_{i}, x_{i+k}\right]\right) .
$$


Note that the postscript font is used $\left(\right.$ range $^{-}$, pessim $\left.^{+}\right)$to emphasize that the quantity is computed, contrary to overestim which is purely theoretical.

The key idea of the algorithm relies on the following simple observation. Any interval $[y]$ included in $[x]$ satisfies at least one of the three conditions:

1. $[y] \subseteq\left[x_{1}, x_{n}\right]$

2. $[y] \subseteq\left[x_{2}, x_{n+1}\right]$

3. $\left[x_{2}, x_{n}\right] \subseteq[y]$

Assume by induction that we know how to bound overestim $[y]$ for any $[y]$ that enter in the two first cases, then, only the third case is left to be treated.

Let us now focus on the third case. We have $\left[x_{2}, x_{n}\right] \subseteq[y] \subseteq\left[x_{1}, x_{n+1}\right]$.

On the one hand,

$$
\operatorname{range}^{-}\left(\left[x_{2}, x_{n}\right]\right) \subseteq f\left(\left[x_{2}, x_{n}\right]\right) \subseteq f([y]) .
$$

On the other hand, by inclusion isotonicity,

$$
F[y] \subseteq F\left[x_{1}, x_{n+1}\right] .
$$

Note that both inclusions are precise if the subdivision is fine, i.e., if $n$ is large. It follows from the two previous inclusions that

$$
\begin{aligned}
\text { overestim }[y] & =\operatorname{rad} F[y]-\operatorname{rad} f([y]) \\
& \leq \operatorname{rad} F\left[x_{1}, x_{n+1}\right]-\operatorname{rad} \operatorname{range}^{-}\left(\left[x_{2}, x_{n}\right]\right) .
\end{aligned}
$$

Therefore, if we know how to estimate the pessimism for $(n-1)$-blocks, we know how to estimate the pessimism for $n$-blocks, i.e., $[x]$. This recursive property implies a combinatory but, fortunately, this particular type of combinatory can be tackled by dynamic programming (see $[3,1]$ ) in polynomial time. More precisely, the worst-case time complexity is in fact $O\left(n^{2}\right)$, and the space complexity is $O(n)$. We just have to start with 1-blocks and 2-blocks, and then proceed to blocks of greater size by applying the idea given above.

Let us now detail the algorithm. We define two $n$-sized arrays denoted by $P^{+}$ (for $\mathbf{P}$ essimism upper estimation), and $R^{-}$(for $\mathbf{R}$ ange inner estimation). $P^{+}$is an array of reals while $R^{-}$is an array of intervals.

After the $k^{t h}$ turn in the outer loop of pessim ${ }^{+}([x], n)$ :

- $P^{+}[i]$ contains pessim ${ }^{+}\left(\left[x_{i}, x_{i+k}\right], k\right)$,

$-R^{-}[i]$ contains range ${ }^{-}\left(\left[x_{i}, x_{i+k-1}\right]\right)$.

Notice that the block size is shifted by 1 in the invariant related to $R^{-}$. This explains why 1-blocks does not require an initialization in the algorithm. 


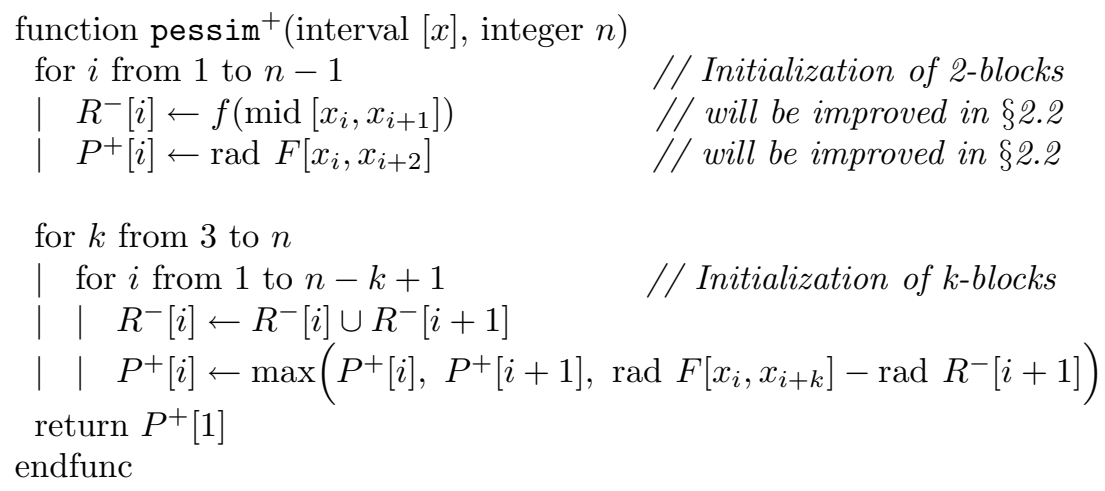

\subsection{Properties of the pessimism upper bound}

Proposition 2. Let $F$ be a Lipschitz-continuous inclusion function that satisfies inclusion isotonicity (i.e., $[x] \subseteq[y] \Longrightarrow F[x] \subseteq F[y]$ ).

The pessimism estimation tends to the actual maximal overestimation when the subdivision size tends to infinity, i.e.,

$$
\operatorname{pessim}^{+}([x], n) \underset{n \rightarrow \infty}{\longrightarrow} \max _{[y] \subseteq[x]} \text { overestim }[y] .
$$

Proof. We already know that for any $n$,

$$
\operatorname{pessim}^{+}([x], n) \geq \max _{[y] \subseteq[x]} \text { overestim }[y] .
$$

We need to prove that $\forall \varepsilon>0$, there exists $n_{0}$ such that for any $n \geq n_{0}$,

$$
\text { pessim }{ }^{+}([x], n)-\max _{[y] \subseteq[x]} \text { overestim }[y]<\varepsilon,
$$

or, in other words, that

$$
\exists[y] \subseteq[x] \operatorname{pessim}^{+}([x], n)-\text { overestim }[y]<\varepsilon .
$$

Let us fix $\varepsilon>0$.

First of all, since a continuous function on a closed interval is also uniformly continuous, there exists $n_{0}$ such that

$$
\forall n \geq n_{0}, \forall x, y \in[x], \quad|x-y| \leq 2 / n \operatorname{rad}[x] \Longrightarrow|f(x)-f(y)|<\varepsilon / 2 .
$$

Furthermore, we can chose $n_{0}$ sufficiently large so that

$$
n_{0} \geq 2 \lambda_{f}([x]) / \varepsilon
$$

is also satisfied. We take now $n \geq n_{0}$, and prove (2) by induction. Base case: $\forall i \in[1, n-1]$, Proposition 1 implies

$$
F\left[x_{i}, x_{i+2}\right] \leq \lambda_{f}([x]) 2 / n<\varepsilon
$$


so that for every 2-block $\left[x_{i}, x_{i+2}\right]$ in $[x]$ we have (by inclusion isotonicity)

$$
\exists[y] \subseteq\left[x_{i}, x_{i+2}\right] \operatorname{pessim}^{+}\left(\left[x_{i}, x_{i+2}\right], 2\right)-\text { overestim }[y]<\varepsilon .
$$

Assume by induction that every $k$-block in $[x]$ satisfy (2), i.e.,

$$
\exists[y] \subseteq\left[x_{i}, x_{i+k}\right] \quad \operatorname{pessim}^{+}\left(\left[x_{i}, x_{i+k}\right], k\right)-\text { overestim }[y]<\varepsilon .
$$

We shall prove that the inequality also holds for $(k+1)$. Consider a $(k+1)$-block $\left[x_{i}, x_{i+k+1}\right] \subseteq[x]$. Let us first prove that $\exists[y] \subseteq\left[x_{i}, x_{i+k+1}\right]$ such that

$$
\left(\operatorname{rad} F\left[x_{i}, x_{i+k+1}\right]-\operatorname{rad} \text { range }^{-}\left(\left[x_{i+1}, x_{i+k}\right]\right)\right)-\text { overestim }[y]<\varepsilon .
$$

To this end, consider an arbitrary interval $[y]$ satisfying

$$
\left[x_{i+1}, x_{i+k}\right] \subseteq[y] \subseteq\left[x_{i}, x_{i+k+1}\right] .
$$

On the one hand, by Definition 1 ,

$$
\begin{aligned}
& q\left(F\left[x_{i}, x_{i+k+1}\right], F\left[x_{i+1}, x_{i+k}\right]\right)<\lambda_{f}([x]) q\left(\left[x_{i}, x_{i+k+1}\right],\left[x_{i+1}, x_{i+k}\right]\right) \\
\Longrightarrow & \operatorname{rad} F\left[x_{i}, x_{i+k+1}\right]-\operatorname{rad} F\left[x_{i+1}, x_{i+k}\right]<\lambda_{f}([x]) \times \\
& \left(\operatorname{rad}\left[x_{i}, x_{i+k+1}\right]-\operatorname{rad}\left[x_{i+1}, x_{i+k}\right]\right) \\
\Longrightarrow \operatorname{rad} F\left[x_{i}, x_{i+k+1}\right]-\operatorname{rad} F\left[x_{i+1}, x_{i+k}\right] & <\lambda_{f}([x]) / n
\end{aligned}
$$

so that, with (4),

$$
\operatorname{rad} F\left[x_{i}, x_{i+k+1}\right]-\operatorname{rad} F\left[x_{i+1}, x_{i+k}\right]<\varepsilon / 2
$$

which implies

$$
\operatorname{rad} F\left[x_{i}, x_{i+k+1}\right]-\operatorname{rad} F[y]<\varepsilon / 2 .
$$

On the other hand, let us denote by $y^{+}$the point where $f$ is maximized in $[y] \subseteq\left[x_{i}, x_{i+k+1}\right]$. The distance between $y^{+}$and the closest point to $y^{+}$among the midpoints of all 1-blocks included in $\left[x_{i+1}, x_{i+k}\right]$ is necessarily less than $2 / n \operatorname{rad}[x]$. The same holds for the point $y^{-}$where $f$ is minimized. Then, thanks to (3), we have

$$
f\left(y^{+}\right)-u b\left(\text { range }^{-}\left(\left[x_{i+1}, x_{i+k}\right]\right)\right)<\varepsilon / 2 \quad(u b \text { stands for "upper bound" })
$$

and

$$
l b\left(\text { range }^{-}\left(\left[x_{i+1}, x_{i+k}\right]\right)\right)-f\left(y^{-}\right)<\varepsilon / 2 \quad(l b \text { stands for "lower bound") },
$$

which means that

$$
\operatorname{rad} f([y])-\operatorname{rad} \operatorname{range}^{-}\left(\left[x_{i+1}, x_{i+k}\right]\right)<\varepsilon / 2 .
$$


Now,

$$
\begin{aligned}
& \left(\operatorname{rad} F\left[x_{i}, x_{i+k+1}\right]-\operatorname{rad} \operatorname{range}^{-}\left(\left[x_{i+1}, x_{i+k}\right]\right)\right)-\text { overestim }[y] \\
= & \left(\operatorname{rad} F\left[x_{i}, x_{i+k+1}\right]-\operatorname{rad} \operatorname{range}^{-}\left(\left[x_{i+1}, x_{i+k}\right]\right)\right)-(\operatorname{rad} F[y]-\operatorname{rad} f([y])) \\
= & \left(\operatorname{rad} F\left[x_{i}, x_{i+k+1}\right]-\operatorname{rad} F[y]\right)+\left(\operatorname{rad} f([y])-\operatorname{rad} \operatorname{range}^{-}\left(\left[x_{i+1}, x_{i+k}\right]\right)\right) \\
< & \varepsilon \quad(\text { by combining }(8) \text { and }(9)),
\end{aligned}
$$

so that (6) is proven. Finally, by definition,

$$
\begin{aligned}
& \operatorname{pessim}^{+}\left(\left[x_{i}, x_{i+k+1}\right], k+1\right):= \\
& \max \left\{\begin{array}{l}
\text { pessim } \\
\operatorname{pessim}^{+}\left(\left[x_{i}, x_{i+k}\right], k\right) \\
\left.\left.\operatorname{rad} F\left[x_{i}, x_{i+k+1}\right]-x_{i+k+1}\right], k\right)
\end{array}\right. \\
& \operatorname{rad} \operatorname{range}^{-}\left(\left[x_{i+1}, x_{i+k}\right]\right)
\end{aligned}
$$

By applying (5) and (6), there exist $[y]^{(1)},[y]^{(2)}$ and $[y]^{(3)}$ in $\left[x_{i}, x_{i+k+1}\right]$ such that

$$
\left\{\begin{array}{l}
\text { pessim } \\
\text { pessim } \\
\text { pes }\left(\left[x_{i}, x_{i+k}, x_{i+k+1}\right], k\right)-\text { overestim }[y]^{(1)}<\varepsilon \\
\left(\operatorname{rad} F\left[x_{i}, x_{i+k+1}\right]-\operatorname{rad} \text { range }{ }^{-}\left(\left[x_{i+1}, x_{i+k}\right]\right)\right)-\text { overestim }[y]^{(3)}<\varepsilon
\end{array}\right.
$$

Hence, there exists $[y] \subseteq\left[x_{i}, x_{i+k+1}\right]$ such that

$$
\operatorname{pessim}^{+}\left(\left[x_{i}, x_{i+k+1}\right], k+1\right) \text {-overestim }[y]<\varepsilon .
$$

Therefore, the inequality (2) is true for $(k+1)$-blocks, which completes the proof.

\subsection{Some improvements}

Initializations in the first loop of pessim ${ }^{+}$can be made more efficient, without however changing the slow (linear) rate of convergence in general.

First, $R^{-}[i]$ was set to the image of the midpoint of $\left[x_{i}, x_{i+1}\right]$, as an inner approximation of $f$ on this 1-block. Clearly, this approximation is made better (i.e., larger) by introducing more sample points in the corresponding interval. However, the overall accuracy remains linearly dependent of the subdivision size (see the proof of Proposition 2).

Although having an influence on the accuracy, the sampling size is therefore not a decisive parameter. On the contrary, the second improvement we propose can speed up the convergence in some situations. Remember that $P^{+}[i]$ is set to $F\left[x_{i}, x_{i+2}\right]$, as an outer approximation of the pessimism on the $i^{t h} 2$-block.

Assume now that $F$ is defined as the natural extension of some differentiable expression of $f$. The overestimation on an interval can also be upwardly approximated using the two next formulae. Since both formulae are likely to be 
precise with small intervals (i.e., 2-blocks) their use may strongly improve the initialization of $P^{+}$.

First, by Proposition 1,

$$
\operatorname{rad} F[x] \leq \lambda_{F}([x]) \operatorname{rad}[x] .
$$

In the case of the natural inclusion function, the constant $\lambda_{F}([x])$ can be automatically computed (see [9] Th. 2.1.1). Second, by the mean value theorem,

$$
\operatorname{rad} f([x]) \geq<F^{\prime}[x]>[x],
$$

where $\left\langle F^{\prime}[x]>\right.$ denotes the mignitude of an interval evaluation of the derivative. Hence,

$$
\operatorname{rad} F[x] \leq \lambda_{F}([x]) /<F^{\prime}[x]>\operatorname{rad} f([x]) .
$$

and since $\operatorname{rad} f([x]) \leq \operatorname{rad} F[x]$,

$$
\operatorname{rad} F[x]-\operatorname{rad} f([x]) \leq \sigma[x] \operatorname{rad} F[x] \quad \text { with } \quad \sigma[x]:=\left(\frac{\lambda_{F}([x])}{<F^{\prime}[x]>}-1\right) .
$$

It follows that the pessimism on $[x]$ is lower than $\sigma[x] \operatorname{rad} F[x]$ and $P^{+}[i]$ should rather be initialized as follows:

$$
P^{+}[i] \leftarrow \max (\sigma[x], 1) \times(\operatorname{rad} F[x]) .
$$

In some cases, this simple trick makes the algorithm behavior more satisfactory. Consider the function $f(x)=x$ and the natural inclusion function $F[x]=[x]$, which is minimal. If the pessimism on each 2 -block $\left[x_{i}, x_{i+2}\right]$ is identified to rad $F\left[x_{i}, x_{i+2}\right]$, the returned value is $2 / n$. The linear rate of convergence prevents from getting an accurate bound. With the help of (12), the best bound (i.e., 0 ) is immediately obtained since $\lambda_{F}([x])=<F^{\prime}[x]>=1$.

\section{Pessimism lower bound}

An algorithm for downwardly approximating the pessimism is built with a very similar induction-based reasoning. Consider a subdivision of $[x]$ with the same notations as before. In the case of $(n+1)$-blocks, if $\left[x_{2}, x_{n}\right] \subseteq[y] \subseteq\left[x_{1}, x_{n+1}\right]$ then

$$
\begin{aligned}
\text { overestim }[y] & =\operatorname{rad} F[y]-\operatorname{rad} f([y]) \\
& \geq \operatorname{rad} F\left[x_{2}, x_{n}\right]-\operatorname{rad} \operatorname{range}^{+}\left(\left[x_{1}, x_{n+1}\right]\right)
\end{aligned}
$$

where range ${ }^{+}$stands for an outer approximation of the range of $f$.

In the following algorithm, $P^{-}[i]$ is a lower bound of the pessimism of $F$ on the $i^{\text {th }} k$-block $\left[x_{i}, x_{i+k}\right]$, while $R^{+}[i]$ contains an outer approximation of the range of $f$ on the same block.

The base case (2-blocks) is handled as follows. 
- $R^{+}[i]$ is initialized with an union of 1-blocks image by $F$. It could also be initialized with the image of the $i^{\text {th }}$ 2-block by any (sharp) inclusion function.

$-P^{-}[i]$ is set to the overestimation on one particular interval, which is the $i^{\text {th }}$ 2-block itself. Again, this bound could be improved by considering a multiplicity of subintervals instead.

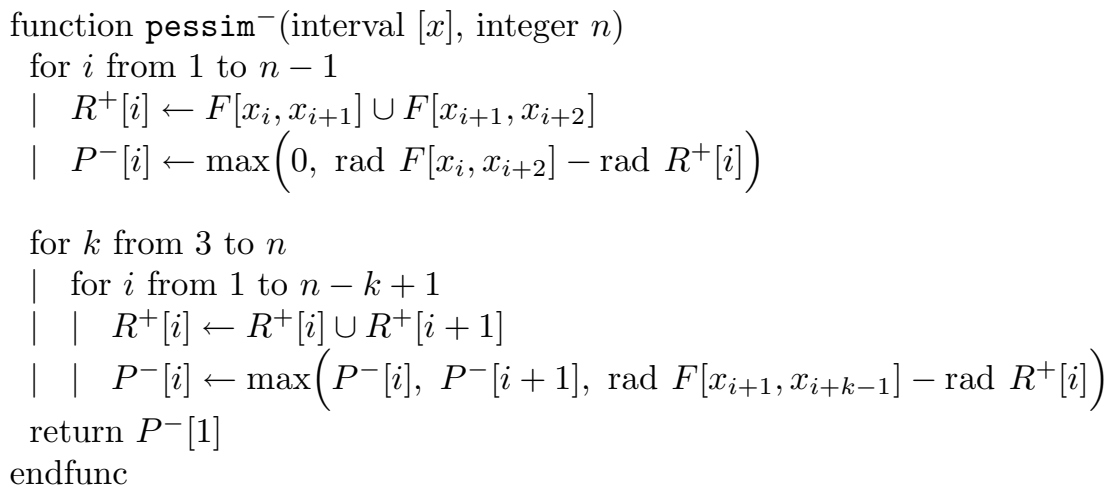

\subsection{Properties of the pessimism lower bound}

Proposition 3. Under the assumptions of Proposition 2, we have

$$
\operatorname{pessim}^{-}([x], n) \underset{n \rightarrow \infty}{\longrightarrow} \max _{[y] \subseteq[x]} \text { overestim }[y]
$$

Proof. The proof being close to Proposition 2's, only a sketch will be given. As before, we prove that $\forall \varepsilon>0$, there exists $n_{0}$ such that $\forall n \geq n_{0}$ and $\forall k \leq n$ $(k \geq 2)$,

$$
\forall i \leq n-k+1 \quad \exists[y] \subseteq\left[x_{i}, x_{i+k}\right] \quad \text { overestim }[y]-\operatorname{pessim}^{-}\left(\left[x_{i}, x_{i+k}\right], k\right)<\varepsilon .
$$

Let us fix $\varepsilon>0$ and consider again $n \geq n_{0}$ where $n_{0}$ satisfies (3) and (4) except that $\varepsilon / 2$ is replaced by $\varepsilon / 3$.

Base case $(k=2)$. Since $\forall i \leq n-1$,

$$
0 \leq \operatorname{pessim}^{-}\left(\left[x_{i}, x_{i+2}\right], 2\right) \leq \operatorname{overestim}\left(\left[x_{i}, x_{i+2}\right]\right) \leq \operatorname{rad} F\left(\left[x_{i}, x_{i+2}\right]\right)<\varepsilon / 3
$$

then $(13)$ is verified with $[y]:=\left[x_{i}, x_{i+2}\right]$.

Induction step $(k+1)$. We consider again an arbitrary interval $[y]$ satisfying

$$
\left[x_{i+1}, x_{i+k}\right] \subseteq[y] \subseteq\left[x_{i}, x_{i+k+1}\right] .
$$

On the one hand, (7) also implies

$$
\operatorname{rad} F[y]-\operatorname{rad} F\left[x_{i+1}, x_{i+k}\right]<\varepsilon / 3 .
$$


On the other hand, the distance between the points $y^{+}$and $x^{+}$where $f$ is maximized over $[y]$ and $\left[x_{i}, x_{i+k+1}\right]$ respectively is necessarily less than $2 / n \operatorname{rad}[x]$. The same distance property holds for the points $y^{-}$and $x^{-}$minimizing $f$. Hence,

$$
\operatorname{rad} f\left(\left[x_{i}, x_{i+k+1}\right]\right)-\operatorname{rad} f([y])<\varepsilon / 3 .
$$

Furthermore,

$$
\text { range }^{+}\left(\left[\mathrm{x}_{\mathrm{i}}, \mathrm{x}_{\mathrm{i}+\mathrm{k}+1}\right]\right) \subseteq \bigcup_{j=i}^{\mathrm{i}+\mathrm{k}} \mathrm{F}\left[\mathrm{x}_{\mathrm{j}}, \mathrm{x}_{\mathrm{j}+1}\right] .
$$

Since $\forall j, i \leq j \leq i+k$, overestim $\left(\left[x_{j}, x_{j+1}\right]\right) \leq \operatorname{rad} F\left[x_{j}, x_{j+1}\right]<\varepsilon / 3$ then

$$
\operatorname{rad} \text { range }{ }^{+}\left(\left[x_{i}, x_{i+k+1}\right]\right)-\operatorname{rad} f\left(\left[x_{i}, x_{i+k+1}\right]\right)<\varepsilon / 3 .
$$

Now,

$$
\begin{aligned}
& \text { overestim }[y]-\left(\operatorname{rad} F\left[x_{i+1}, x_{i+k}\right]-\operatorname{rad} \operatorname{range}^{+}\left(\left[x_{i}, x_{i+k+1}\right]\right)\right) \\
= & (\operatorname{rad} F[y]-\operatorname{rad} f([y]))-\left(\operatorname{rad} F\left[x_{i+1}, x_{i+k}\right]-\operatorname{rad} f\left(\left[x_{i}, x_{i+k+1}\right]\right)+\right. \\
& \left.\operatorname{rad} f\left(\left[x_{i}, x_{i+k+1}\right]\right)-\operatorname{rad} \operatorname{range}^{+}\left(\left[x_{i}, x_{i+k+1}\right]\right)\right) \\
= & \left(\operatorname{rad} F[y]-\operatorname{rad} F\left[x_{i+1}, x_{i+k}\right]\right) \\
& +\left(\operatorname{rad} \operatorname{range}{ }^{+}\left(\left[x_{i}, x_{i+k+1}\right]\right)-\operatorname{rad} f\left(\left[x_{i}, x_{i+k+1}\right]\right)\right) \\
& +\left(\operatorname{rad} f\left(\left[x_{i}, x_{i+k+1}\right]\right)-\operatorname{rad} f([y])\right) \\
< & \varepsilon \quad(\operatorname{bycombining}(14),(15) \text { and }(16))
\end{aligned}
$$

The end of the proof is left to the reader.

\section{Relative pessimism}

One could find more relevant to compute the relative pessimism, i.e.,

$$
\max _{[y] \subseteq[x], \operatorname{rad} f([y]) \neq 0} \operatorname{rad} F[y] / \operatorname{rad} f([y]) .
$$

Our approach can be adapted for such a purpose. We shall give a brief overview of this variant. The induction step resorts naturally to the following inequalities: if $\left[x_{2}, x_{n}\right] \subseteq[y] \subseteq\left[x_{1}, x_{n+1}\right]$ then

$$
\frac{\operatorname{rad} F\left[x_{2}, x_{n}\right]}{\operatorname{rad} \operatorname{range}^{+}\left(f,\left[x_{1}, x_{n+1}\right]\right)} \leq \frac{\operatorname{rad} F[y]}{\operatorname{rad} f([y])} \leq \frac{\operatorname{rad} F\left[x_{1}, x_{n+1}\right]}{\operatorname{rad} \operatorname{range}^{-}\left(f,\left[x_{2}, x_{n}\right]\right)}
$$

Let us focus on the base case. For an upper approximation of the relative pessimism, the base case can be tackled thanks to (10). For the lower approximation, we need to introduce a new formula. Notice that, with Proposition 1 in view, 
the Lipschitz constant $\lambda_{F}([x])$ can be defined recursively by applying classical formulae like:

$$
\begin{aligned}
& \operatorname{rad}([x] \pm[y]) \leq \operatorname{rad}[x]+\operatorname{rad}[y], \\
& \operatorname{rad}([x] \times[y]) \leq|[y]| \operatorname{rad}[x]+|[x]| \operatorname{rad}[y], \\
& \operatorname{rad} \phi([x]) \leq\left|\phi^{\prime}([x])\right| \operatorname{rad}[x], \\
& \text {... }
\end{aligned}
$$

It happens that inequality signs can be reversed by substituting mignitudes to magnitudes:

$$
\begin{aligned}
& \operatorname{rad}([x] \pm[y]) \geq \operatorname{rad}[x]+\operatorname{rad}[y], \\
& \operatorname{rad}([x] \times[y]) \geq<[y]>\operatorname{rad}[x]+<[x]>\operatorname{rad}[y], \\
& \operatorname{rad} \phi([x]) \\
& \ldots<\phi^{\prime}([x])>\operatorname{rad}[x],
\end{aligned}
$$

Hence, one can also compute for any interval $[x]$ a constant $\mu_{F}([x])$ that verifies

$$
\operatorname{rad} F([x]) \geq \mu_{F}([x]) \operatorname{rad}[x],
$$

so that the base case can be handled with

$$
\frac{\mu_{F}([y])}{\left|F^{\prime}[y]\right|} \leq \frac{\operatorname{rad} F[y]}{\operatorname{rad} f([y])} \leq \frac{\lambda_{F}([y])}{<F^{\prime}[y]>} .
$$

\section{Pessimism control}

Now, as we know how to estimate the pessimism, the natural next step is to control the pessimism of an inclusion function over a (possibly large) initial interval $[x]$. More precisely, the goal is to split $[x]$ into sub-intervals where the pessimism is guaranteed to meet some requirements.

Such a question needs however some precaution, simply because computing the pessimism does not cancel the pessimism. Consider for instance the function $f(x)=x-x$ and the natural inclusion function $F[x]=[x]-[x]$. If one requires the pessimism to be lower than $10^{-5}$ in $[x]=[0,1]$, the best algorithm ever would anyway yield $2 \times 10^{5}$ intervals since in this case the overestimation of $F[x]$ is always $2 \times \operatorname{rad}[x]$.

What follows must therefore be applied in situations where the requirements are "compatible" with the inclusion function.

Furthermore, the algorithm should detect blocks (i.e., sub-intervals) for which the pessimism is small enough, instead of returning a single value corresponding to the pessimism for the overall interval. This would avoid redundant computations in a test and bisect strategy.

The goal is to adapt the pessim ${ }^{+}$algorithm in such a way that a sequence of "accurate" blocks is returned instead of the overall pessimism: each block in this sequence is either "accurate" or a 1-block $\left[x_{i}, x_{i+1}\right]$ that has to be further analyzed. We must generate a sequence of minimal size and keep the quadratic 
complexity at the same time. To this end, we introduce an extra array $B$ (for Block) of integers. In return, $B[i]$ shall contain the size $k$ of the largest "accurate" block starting from $x_{i}$ (and 0 if none).

The algorithm pessim ${ }^{+}$can then be reimplemented as follows:

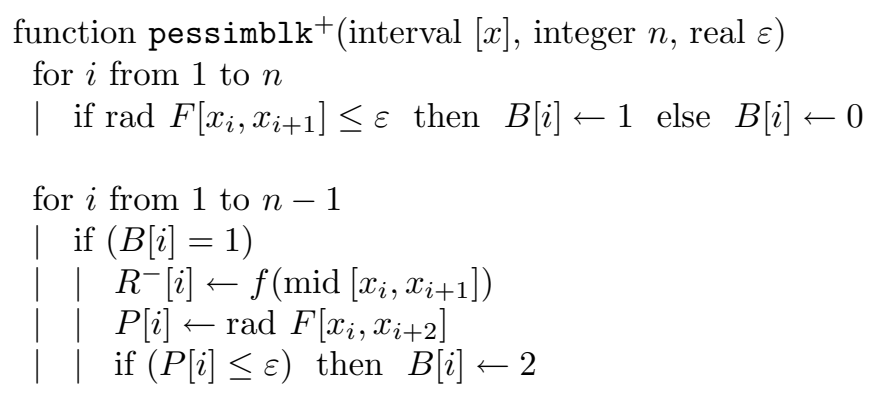

for $k$ from 3 to $n$

| for $i$ from 1 to $n-k+1$

| $\quad$ if $(B[i]=B[i+1]=k-1)$

| | $\quad R^{-}[i] \leftarrow R^{-}[i] \cup R^{-}[i+1]$

| | if $\left(\operatorname{rad} F\left[x_{i}, x_{i+k}\right]-\operatorname{rad} R^{-}[i+1]\right) \leq \varepsilon$ then $B[i] \leftarrow k$

return $B$

endfunc

The pessim-ctrl algorithm can finally be given. This algorithm generates a sequence of consecutive intervals that forms a partition ${ }^{2}$ of the input domain $[x]$. On each interval of this partition, the inclusion function $F$ is proven to be accurate (according to our absolute criterion $\varepsilon$ ). After a call to pessimblk ${ }^{+}$, the array $B$ is read forward and pessim-ctrl is only called recursively with 1-blocks where $F$ is not accurate. Hence, no computation is redundant since 1blocks are precisely those which are not subdivided by the pessimblk ${ }^{+}$function.

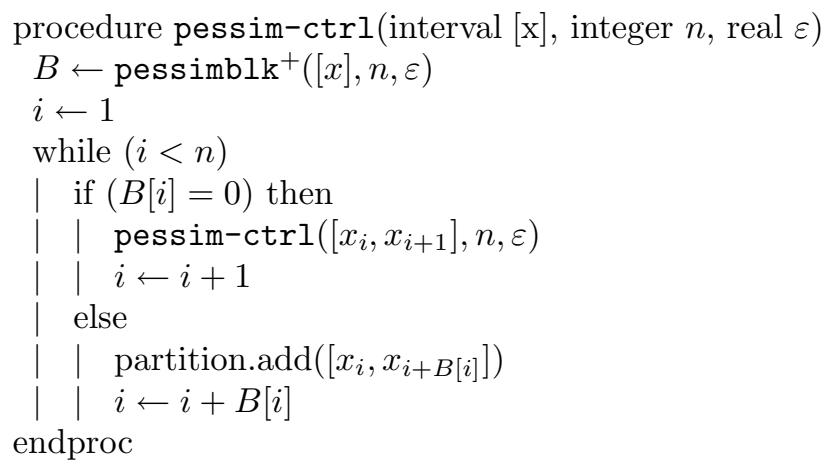

\footnotetext{
${ }^{2}$ For simplicity, we store here the partition in a simple list named partition. However, a tree structure might sometimes be more adapted for practical uses.
} 


\section{First experiments}

Let us first illustrate numerically the behavior of pessim ${ }^{+}$and pessim ${ }^{-}$(both are merged into a unique algorithm). We have considered $f(x)=\sin (x)+x$ and $F[x]=\sin [x]+[x]$ with $[x]=[0,10]$. Results obtained are summed up in the following table. We can check that the precision goes linearly with the subdivision size $n$ while the computation time goes quadratically (notice that $n$ grows exponentially). The program has been run on a standard laptop. The upper and lower approximation seem to converge towards 2.0 which is the actual pessimism of $F$ on $[x]$ : the interval with the maximal overestimation 2.0 being $[\pi / 2,3 \pi / 2]$.

\begin{tabular}{|r|l|l|l|}
$\mathrm{n}$ & pessim $^{-}$ & pessim $^{+}$ & time (in sec) \\
\hline 2 & 0. & 6. & $\sim 0$ \\
4 & 0.700764 & 4.2787 & $\sim 0$ \\
8 & 0.799236 & 3.20395 & $\sim 0$ \\
16 & 1.27016 & 2.58151 & 0.004 \\
32 & 1.6413 & 2.31233 & 0.004 \\
64 & 1.83253 & 2.15608 & 0.020001 \\
128 & 1.91915 & 2.07795 & 0.060004 \\
256 & 1.96015 & 2.039 & 0.216013 \\
512 & 1.98035 & 2.01951 & 0.884055 \\
1024 & 1.9902 & 2.00976 & 3.47222 \\
2048 & 1.99511 & 2.00488 & 14.0449 \\
4096 & 1.99756 & 2.00244 & 56.2235 \\
8192 & 1.99878 & 2.00122 & 223.418
\end{tabular}

Table 1. Lower and upper approximation of the pessimism.

Let us deal now with a more sophisticated example. Assume

$$
f(x)=\cos (x) \sin (x)+\cos (x) \exp (-x)+\sin (x) \exp (-x) .
$$

One may wonder which term should be factored out when using interval arithmetic, i.e., which of the following inclusion functions is sharper in general:

$$
\begin{aligned}
& F_{1}[x]=\cos [x] \times(\sin [x]+\exp (-[x]))+\sin [x] \times \exp (-[x]), \\
& \left.F_{2}[x]=\sin [x] \times(\cos [x]+\exp (-[x]))+\cos [x] \times \exp (-[x])\right), \\
& F_{3}[x]=\exp (-[x]) \times(\cos [x]+\sin ([x]))+\cos [x] \times \sin [x] .
\end{aligned}
$$

We assume that intervals $[x]$ in argument always satisfy $[x] \subseteq[0,10]$.

Of course, computing the pessimism of each inclusion function on $[0,10]$ is not really relevant. To make a fair comparison between these inclusion functions, we have run the pessim-ctrl algorithm with different (exponentially decreasing) values for $\varepsilon$. If the partition size (i.e., the number of splits necessary to achieve the 
desired precision) with an inclusion function is always smaller than the partition size with another one, then the first function can be considered (experimentally) as sharper. Of course, this comparison must be interpreted "in average" since for one peculiar interval, we cannot predict which of them entails the biggest overestimation.

Results are depicted in Figure 1.

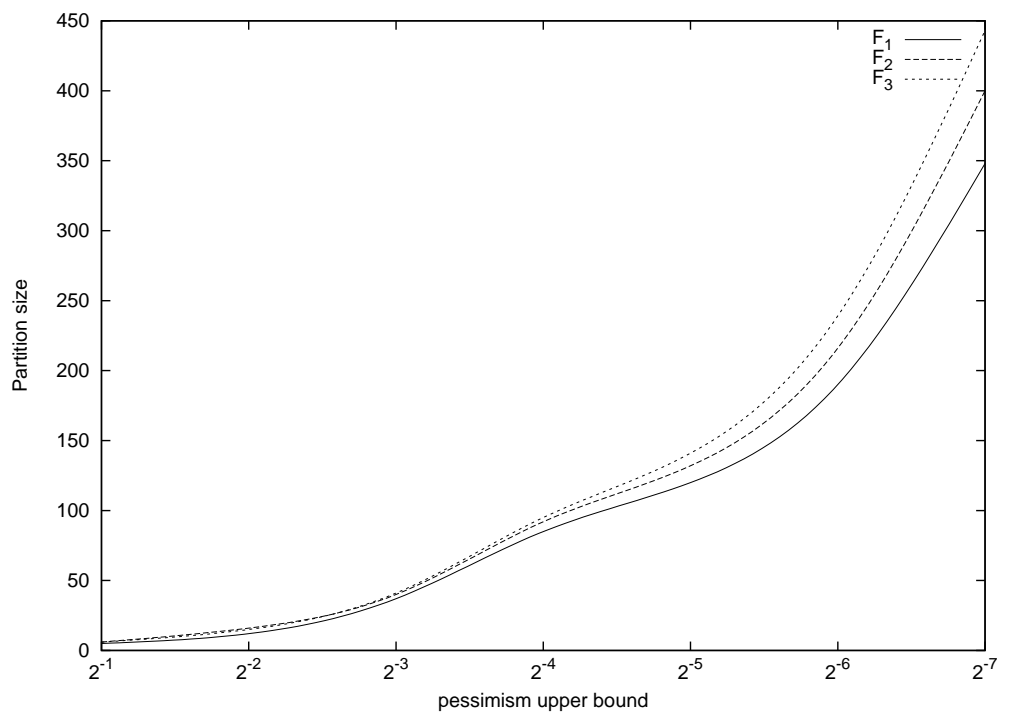

Fig. 1. Number of necessary splits to achieve a desired precision. Each curve is associated to an inclusion function. A curve maps (with interpolated values) the partition size obtained with the pessim-ctrl algorithm to the input parameter (of maximal pessimism) $\varepsilon$. The input interval $[x]$ is set to $[0,10]$. The inclusion function $F_{1}$ seems to be more sharp with any value of $\varepsilon$; the ratio between $F_{1}$ 's values and the others' even decreases with $\varepsilon$ (down to $78 \%$ with $\varepsilon=2^{-7}$ ).

\section{Conclusion}

This paper is a first exploration of a new concept related to inclusion functions, called pessimism computation. We have given algorithms (pessim ${ }^{+}$and pessim ${ }^{-}$) for bounding the overestimation over a infinite set of possible input intervals. These algorithms are valid for any type of (inclusion isotone) inclusion functions. If, in addition, the inclusion function is Lipschitz-continuous (which is most of the time true), the algorithms converge to the optimal result (see Proposition 2 and 3). However, the convergence rate is only linear. 
We have also provided a variant for computing the relative pessimism. Some work is left to be done in this direction. As an example, the algorithm could be modified to deal with singularities and the convergence issue could be investigated.

In a second part, we have adapted the pessim ${ }^{+}$algorithm in order to control the pessimism of the inclusion function, i.e., to automatically decompose a domain into sub-intervals where the pessimism is small enough. The efficiency of the analysis is significantly improved by checking at any stage whether the function is accurate or not (block management), avoiding any redundant computation.

We have also shown that the pessim-ctrl algorithm could be used as a way to compare -in average- the accuracy of different inclusion functions.

All the results we have presented in this paper can easily be adapted to vectorvalued functions. However, extending our work to the multi-variable case seems not straightforward. Such extension would probably resort to multidimensional dynamic programming, which is known to have an exponential complexity. This issue might be addressed in future works.

\section{References}

1. R. Bellman. Dynamic Programming. Princeton University Press, 1957.

2. F. Benhamou, D. McAllester, and P. Van Hentenryck. CLP(intervals) revisited. In International Symposium on Logic programming, pages 124-138. MIT Press, 1994.

3. T.H. Cormen, C.E. Leiserson, and R.L. Rivest. Introduction to Algorithms. MIT Press, Cambridge, 1990.

4. J. Garloff, C. Jansson, and A.P. Smith. Lower bound functions for polynomials. J. Comput. Appl. Math., 157(1):207-225, 2003.

5. L. Jaulin, M. Kieffer, O. Didrit, and E. Walter. Applied Interval Analysis. Springer, 2001.

6. R.B. Kearfott. Rigorous Global Search: Continuous Problems. Springer, 1996.

7. K. Makino and M. Berz. Taylor Models and Other Validated Functional Inclusion Methods. International Journal of Pure and Applied Mathematics, 4(4):379-456, 2003.

8. R. Moore. Interval Analysis. Prentice-Hall, 1966.

9. A. Neumaier. Interval Methods for Systems of Equations. Cambridge University Press, 1990.

10. H. Ratschek and J. Rokne. Computer Methods for the Range of Functions. Halsted Press, 1984.

11. N. Revol and F. Rouillier. Motivations for an arbitrary precision interval arithmetic and the MPFI library. Reliable Computing, 11(4):275-290, 2005.

12. P. Van Hentenryck, L. Michel, and Y. Deville. Numerica: A Modeling Language for Global Optimization. MIT Press, Cambridge, 1997. 